\title{
On the relation between hybrid and pure spinor string theory
}

\author{
Sebastian Gerigk ${ }^{a}$ and Ingo Kirsch ${ }^{b}$ \\ ${ }^{a}$ Institut für Theoretische Physik, ETH Zürich, \\ Wolfgang-Pauli-Strasse 27, CH-8093 Zürich, Switzerland \\ ${ }^{b}$ DESY Hamburg, Theory Group, \\ Notkestrasse 85, D-22607 Hamburg, Germany \\ E-mail: gerigk@phys.ethz.ch, ingo.kirsch@desy.de
}

ABSTRACT: In this paper we revisit Berkovits' pure spinor formalism in lower dimensions. We are particularly interested in relating a six-dimensional pure spinor action previously constructed in the literature to other superstring formalisms. In order to gain some insight into six-dimensional pure spinors, we first derive their action by gauge-fixing the classical six-dimensional Green-Schwarz action. We then consider a hybrid pure spinor construction in which the spacetime symmetries of six of the ten dimensions are described in pure spinor variables, while the remaining four dimensions are parameterized in terms of RNS variables. We relate this pure spinor formalism to the Berkovits-Vafa-Witten hybrid formalism of string theory on $\mathbb{R}^{6} \times T^{4}$.

KeYwords: Conformal Field Models in String Theory, BRST Symmetry

ARXIV EPRINT: 0912.2347 


\section{Contents}

1 Introduction $\quad 1$

2 Green-Schwarz versus pure spinor formalism in six dimensions $\quad 3$

2.1 Pure spinors in six-dimensions 3

2.2 Equivalence of the pure spinor formalism and the Green-Schwarz action in six dimensions

3 From hybrid to pure spinor formalism

3.1 Hybrid formalism with manifest $\mathcal{N}=1$ superspace variables 6

$\begin{array}{ll}3.2 & \text { Gauge-fixed hybrid action }\end{array}$

$\begin{array}{lll}3.3 & \text { Equivalence of the pure spinor and hybrid actions } & 7\end{array}$

$\begin{array}{lll}3.4 & \text { Comments on the BRST operators } & 9\end{array}$

$\begin{array}{ll}\text { A Six-dimensional gamma matrices } & 12\end{array}$

B Summary of fields in RNS, hybrid and pure spinor formalism 12

\section{Introduction}

In the past ten years alternative superstring formalisms have been developed to surmount the shortcomings of the Ramond-Neveu-Schwarz (RNS) and Green-Schwarz (GS) formalisms. The most prominent among them is Berkovits' pure spinor formalism [1-3], see [4-6] for reviews and lectures. As the GS formalism, the pure spinor theory exhibits manifest super Poincaré invariance but in contrast to the former it can be quantised in a straightforward manner.

Naturally one is also interested in compactifications of the pure spinor formalism to lower dimensions and their relation to standard superstring theories. Pure spinor models in $d=2,4,6$ (flat) dimensions were constructed in $[3,7,8]$, see also $[9,10]$ for non-critical pure spinor superstrings. The $d=10$ pure spinor theory has been related to the RNS superstring by twisting the ten spin-half RNS fermions using an $\mathrm{SO}(10) / \mathrm{U}(5)$ pure spinor variable [11]. In the same paper [11] it was also shown that the $d=10$ formalism can be obtained by gauge-fixing the GS superstring. Unlike the $d=10$ case, the relation of lower-dimensional models to known string theories is rather elusive and has not yet been proven.

In this paper we focus on the $d=6$ pure spinor theory in flat space $[7,8]$. In section 2 we show along the lines of [11] that the $d=6$ pure spinor action can be obtained by gauge-fixing the six-dimensional GS action. The latter is known to contain 4 first-class and 4 secondclass constraints. After an appropriate splitting of the ghost variables, the 4 second-class constraints can be converted into 2 first-class constraints, giving 6 first-class constraints in 


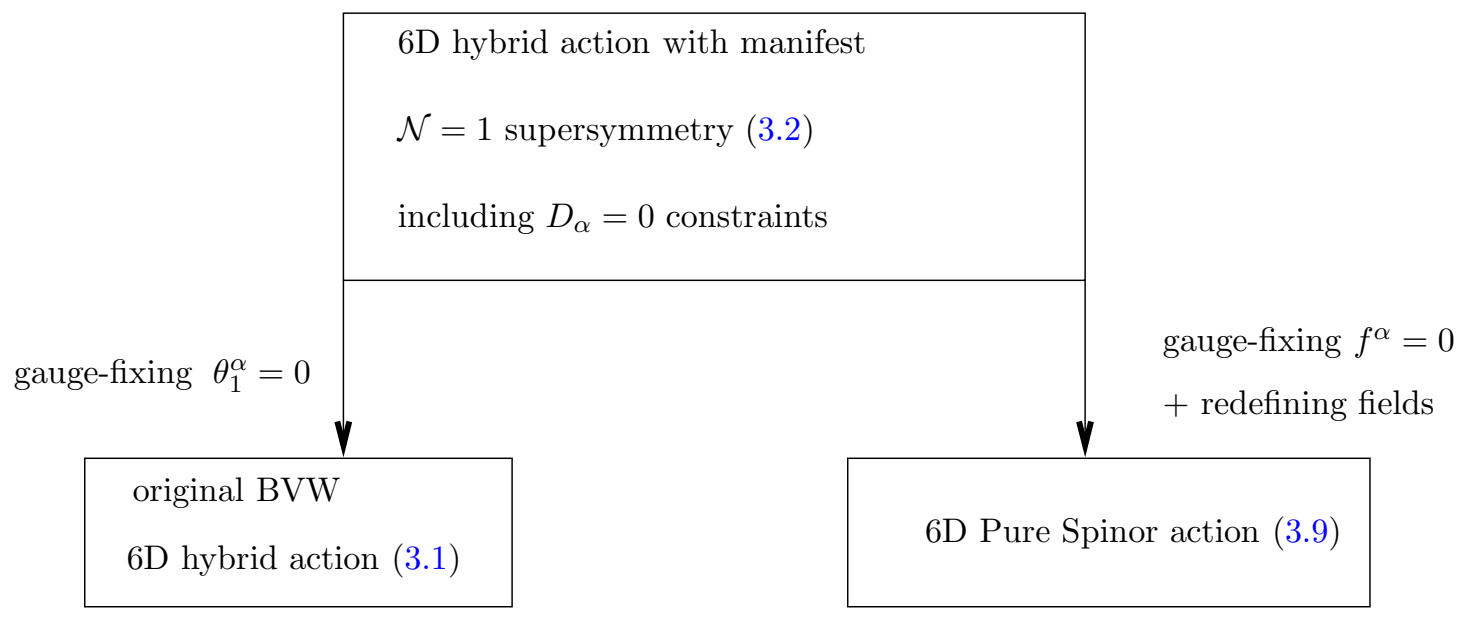

Figure 1. Schematical illustration of the equivalence of the $6 \mathrm{D}$ hybrid formalism and the $6 \mathrm{D}$ pure spinor formalism as introduced in $[7,8]$.

total. Gauge-fixing the corresponding Lagrange multipliers will then introduce six bosonic ghosts. Five of them make up the pure spinor, which has five independent components in six dimensions. After removing the sixth ghost by a similarity transformation, the resulting action becomes the $d=6$ pure spinor action.

In section 3 we then discuss the relation to the six-dimensional Berkovits-Vafa-Witten hybrid formalism for superstring theory on $\mathbb{R}^{6} \times T^{4}$ [12]. For this, we supplement the six-dimensional pure spinor theory of $[7,8]$ with a four-dimensional action for the compactification on the four-torus $T^{4}$. The internal part on $T^{4}$ is formulated in RNS variables and is the same as in the hybrid formalism. We therefore only need to show that the external part on $\mathbb{R}^{6}$ of the hybrid action (plus the action for the chiral bosons $\sigma$ and $\rho$ ) can be replaced by the six-dimensional pure spinor action of $[7,8]$.

In order to show the equivalence of both formalisms, we perform a series of manipulations of the original hybrid action. An overview is given in figure 1, which begins with the original hybrid action (left box) rewritten with manifest six-dimensional supersymmetry (top box) and ends at the pure spinor action (right box). In the original hybrid action only half of the $\mathcal{N}=1$ superspace variables are manifest, i.e. four out of eight $\theta$ variables. We therefore follow [13] and add four further $\theta$ 's and four "harmonic" constraints $D_{\alpha}$ to the action, giving the hybrid action with manifest $\mathcal{N}=1$ supersymmetry (top box). These constraints are first-class and allow the additional $\theta$ 's to be gauged away [13]. Instead of gauging them away, we add the four constraints $D_{\alpha}$ to the action using Lagrange multipliers $f^{\alpha}$. We then gauge-fix the $f^{\alpha}$ to zero, which introduces four bosonic ghost fields. Another ghost field of the same kind comes from the $\sigma$ - $\rho$-sector of the hybrid action. After an appropriate field redefinition, the four plus one ghost fields combine to give the five components of the six-dimensional pure spinor, and the hybrid action turns into the pure spinor action (right box). We also show that the same field transformation maps the BRST operators into each other. We close with some remarks on the cohomology. 


\section{Green-Schwarz versus pure spinor formalism in six dimensions}

In this section we derive the pure spinor action in six dimensions by gauge-fixing the sixdimensional Green-Schwarz action, which is consistent on the classical level. The impatient reader, who is interested only in the connection to the hybrid string, may wish to immediately proceed to section 3 after reading the general introduction on pure spinors in six dimensions in section 2.1.

\subsection{Pure spinors in six-dimensions}

Pure spinors in six dimensions are discussed in detail in $[7,8]$. Here we only review some of their basic properties. Six-dimensional pure spinors are given by two $\mathrm{SO}(6)$ Weyl spinors $\lambda_{I}^{\alpha}(I=1,2 ; \alpha=1, \ldots, 4)$ which are subject to the constraint

$$
\varepsilon^{I J} \lambda_{I}^{\alpha} \Gamma_{\alpha \beta}^{m} \lambda_{J}^{\beta}=0
$$

where the $\Gamma^{m}$ are the antisymmetric $4 \times 4$ off-diagonal blocks of the six-dimensional gamma matrices given in appendix A. They satisfy

$$
\Gamma_{\alpha \beta}^{(m} \Gamma^{n) \beta \gamma}=\eta^{m n} \delta_{\alpha}^{\gamma} .
$$

The corresponding matrices with upper indices are defined by $\Gamma^{m \alpha \beta}=\frac{1}{2} \varepsilon^{\alpha \beta \gamma \delta} \Gamma_{\gamma \delta}^{m}$.

We may now go to light-cone gauge by defining $\Gamma^{ \pm}=\Gamma^{0} \pm \Gamma^{5}$. This induces a symmetry breaking of $\mathrm{SO}(6)$ down to $\mathrm{SO}(4)$ under which the Weyl spinor representation $\mathbf{4}_{\mathbf{S}}$ decomposes into $\mathbf{2}_{\mathbf{s}} \oplus \mathbf{2}_{\mathbf{c}}$. Explicitly, the spinor can be solved by writing $\lambda_{I}^{\alpha}=\left(\lambda_{I}^{A}, \lambda_{I}^{\dot{A}}\right)$ with $A, \dot{A}=1,2$. The pure spinor constraint (2.1) then decomposes into

$$
\varepsilon^{I J} \lambda_{I}^{A} \varepsilon_{A B} \lambda_{J}^{B}=0, \quad \varepsilon^{I J} \lambda_{I}^{\dot{A}} \varepsilon_{\dot{A} \dot{B}} \lambda_{J}^{\dot{B}}=0, \quad \varepsilon^{I J} \lambda_{I}^{A} \lambda_{J}^{\dot{B}}=0 .
$$

As shown in [7], the second and third constraints are solved by

$$
\lambda_{I}^{\dot{B}}=h_{(0)}^{\dot{B}} A \lambda_{I}^{A}
$$

provided the first constraint is satisfied. The field $h_{(0)}^{\dot{B}}$ consists of four degrees of freedom. However, there is an infinite number of gauge symmetries $(n=0,1, \ldots)$

$$
\delta h_{(n)}^{\dot{B}} A=\varepsilon^{I J} \varepsilon_{A B} \eta_{(n) I}^{\dot{B}} \lambda_{J}^{B}, \quad \delta \eta_{(n) I}^{\dot{B}}=h_{(n+1)}^{\dot{B}} \lambda_{I}^{A},
$$

which reduce the number of degrees of freedom of $\lambda_{I}^{\dot{B}}$ to $4-4+4-4+\cdots=4 \sum_{k}(-1)^{k}=2$. At the same time the first constraint reduces the number of degrees of freedom of $\lambda_{I}^{A}$ to three. The six-dimensional pure spinor therefore has only five independent components.

\subsection{Equivalence of the pure spinor formalism and the Green-Schwarz action in six dimensions}

For $\mathcal{N}=(1,0)$ supersymmetry in $d=6$ the left-moving (holomorphic) matter worldsheet fields are $\left(x^{m}, \theta_{I}^{\alpha}, p_{\alpha}^{I}\right)$, where $\theta_{I}^{\alpha}$ is a doublet $(I=1,2)$ of four-component Weyl spinors, and $p_{\alpha}^{I}$ are their conjugate momenta $(\alpha=1, \ldots, 4)$. 
The six-dimensional Green-Schwarz action in first-order form is given by

$$
S=\int d^{2} z\left[\frac{1}{2} \partial x^{m} \bar{\partial} x_{m}+p_{\alpha}^{I} \bar{\partial} \theta_{I}^{\alpha}-e\left(\frac{1}{2} \partial x^{m} \partial x_{m}+p_{\alpha}^{I} \partial \theta_{I}^{\alpha}\right)\right],
$$

where $m=0, \ldots, 5$. Since the action is in conformal gauge, the Virasoro constraint $T=0$ has been added to the action using a Lagrange multiplier $e$. The action is also supplemented by the constraints

$$
d_{\alpha}^{I}=p_{\alpha}^{I}-\frac{1}{2} \varepsilon^{I J}\left(\Gamma_{m} \theta_{J}\right)_{\alpha}\left(\partial x^{m}+\frac{1}{4} \varepsilon^{K L}\left(\theta_{K} \Gamma^{m} \partial \theta_{L}\right)\right) .
$$

Setting $d_{\alpha}^{I}=0$ and substituting the resulting equation for the conjugate momentum $p_{\alpha}^{I}$ back into (2.6) yields the standard form of the Green-Schwarz action.

Using the identity (A.1), the constraints (2.7) can be rewritten as

$$
d_{\alpha}^{I}=p_{\alpha}^{I}-\frac{1}{2} \varepsilon^{I J}\left(\Gamma_{m} \theta_{J}\right)_{\alpha} \partial x^{m}-\frac{1}{4} \varepsilon^{I J} \varepsilon^{K L} \varepsilon_{\alpha \beta \gamma \delta} \theta_{J}^{\beta} \theta_{K}^{\gamma} \partial \theta_{L}^{\delta} .
$$

They satisfy the OPE [7]

$$
d_{I \alpha}(z) d_{J \beta}(w)=-(z-w)^{-1} \varepsilon_{I J} \not \prod_{\alpha \beta},
$$

where $\llbracket=\Pi_{m} \Gamma^{m}$. Since $\bigsqcup^{2}=0, d_{\alpha}^{I}$ separate into four first-class and four second-class constraints, denoted by $d_{A}^{I}$ and $d_{\dot{A}}^{I}$, respectively.

We now incorporate the GS-constraints (2.8) into the action (2.6) by introducing Lagrange multiplier terms $f_{I}^{\alpha} d_{\alpha}^{I}$. The first-class constraints can be eliminated by gauge-fixing $f_{I}^{A}=0$ in $f_{I}^{\alpha} d_{\alpha}^{I}=f_{I}^{A} d_{A}^{I}+f_{I}^{\dot{A}} d_{\dot{A}}^{I}$. This introduces four $\beta$ - $\gamma$-systems with weights $(1,0)$, denoted by $\beta_{A}^{I}$ and $\gamma_{I}^{A}$. Introducing also the usual gauge-fixing term $b \bar{\partial} c$ for $e=0$, we get

$$
S=\int d^{2} z\left[\frac{1}{2} \partial x^{m} \bar{\partial} x_{m}+p_{\alpha}^{I} \bar{\partial} \theta_{I}^{\alpha}+f_{I}^{\dot{A}} d_{\dot{A}}^{I}+b \bar{\partial} c+\beta_{A}^{I} \bar{\partial} \gamma_{I}^{A}\right]
$$

Next, we need to express the four second-class constraints $d_{\dot{A}}^{I}$ in terms of two firstclass constraints. For this, we regroup the ghosts $\gamma_{I}^{A} \rightarrow\left(\tilde{\gamma}, \lambda_{I}^{A}\right)$ into $\lambda_{I}^{A}$, which is subject to the first constraint of (2.3) and therefore has three independent components, and one component $\tilde{\gamma}$. We then define the first-class constraints

$$
H^{M} \equiv \varepsilon^{I J} \lambda_{I}^{\alpha}\left(\Gamma^{-} \Gamma^{M}\right)_{\alpha \dot{\alpha}} d_{J}^{\dot{\alpha}}=\varepsilon^{I J} \lambda_{I}^{A} \sigma_{A \dot{A}}^{M} d_{J}^{\dot{A}}
$$

with $M=1,2,3,4$. Here we used that the matrices $\Gamma^{-} \Gamma^{M}$ are $4 \times 4$ are matrices of the type

$$
\left(\begin{array}{cc}
0 & \sigma^{M} \\
0 & 0
\end{array}\right),
$$

where $\sigma^{M}$ are the standard $2 \times 2$ Pauli matrices (with one of them the identity operator $\mathbf{1}_{2}$ ). As the four $\sigma^{M}$-matrices give a basis of $2 \times 2$ matrices, the constraints in eq. (2.11) are equivalent to the four constraints

$$
\epsilon^{I J} \lambda_{I}^{A} d_{J}^{\dot{A}}=0
$$


These can be solved by

$$
d_{J}^{\dot{A}}=M_{(0) \dot{B}}^{\dot{A}} \lambda_{J}^{\dot{B}}
$$

for any linear mapping $M$, which has four degrees of freedom. However, as in (2.4) and (2.5), there is an infinite number of gauge symmetries which reduce the number of degrees of freedom to two. Thus, only two of the four constraints $H^{M}$ are independent.

We may therefore write $f_{I}^{\dot{A}} d_{\dot{A}}^{I}=h_{M} H^{M}$, where only two of the four Lagrange multipliers $h_{M}$ are non-vanishing, say those for $M=0,1$. Gauge-fixing them to zero yields two further bosonic $\beta$ - $\gamma$-systems which we denote by $w^{M}$ and $\lambda_{M}$ (now e.g. $M=0,1$ only). The action is then

$$
S=\int d^{2} z\left[\frac{1}{2} \partial x^{m} \bar{\partial} x_{m}+p_{\alpha}^{I} \bar{\partial} \theta_{I}^{\alpha}+w^{M} \bar{\partial} \lambda_{M}+b \bar{\partial} c+w_{A}^{I} \bar{\partial} \lambda_{I}^{A}+\tilde{\beta} \bar{\partial} \tilde{\gamma}\right] .
$$

Here the last two terms descend from the last term in (2.10) according to the decompositions $\gamma_{I}^{A} \rightarrow\left(\tilde{\gamma}, \lambda_{I}^{A}\right)$ and $\beta_{A}^{I} \rightarrow\left(\tilde{\beta}, w_{A}^{I}\right)$.

The spinors $\lambda^{M}$ (two degrees of freedom) and $\lambda_{I}^{A}$ (three degrees of freedom) then make up the pure spinor $\lambda_{I}^{\alpha}$ (five independent components). Note that the two degrees of freedom of $\lambda^{M}$ can be rearranged into the pure spinor components $\lambda_{I}^{\dot{A}}$, which has also two independent components, as shown in section 2.1. As in the ten-dimensional case [11], an appropriate similarity transformation of the action removes the ghost terms $b \bar{\partial} c$ and $\tilde{\beta} \bar{\partial} \tilde{\gamma}$. The action can then be written in terms of the pure spinor $\lambda_{I}^{\alpha}$ as

$$
S=\int d^{2} z\left[\frac{1}{2} \partial x^{m} \bar{\partial} x_{m}+p_{\alpha}^{I} \bar{\partial} \theta_{I}^{\alpha}+w_{\alpha}^{I} \bar{\partial} \lambda_{I}^{\alpha}\right],
$$

which is the six-dimensional pure spinor action of $[7,8]$.

Let us finally determine the central charges. The pure spinor fields $w, \lambda$ formally represent five (bosonic) $\beta$ - $\gamma$-systems with weights $(1,0)$. The fermions $p, \theta$ correspond to eight (fermionic) $b$-c-systems of weight $(1,0)$. The central charges are therefore $c_{w, \lambda}=$ $5 \cdot 2=10, c_{p, \theta}=8 \cdot(-2)=-16$ and $c_{x}=6$. The total central charge is thus zero.

\section{From hybrid to pure spinor formalism}

In this section we derive the pure spinor action in six dimensions $[7,8]$ from the BerkovitsVafa-Witten hybrid formalism for string theory on $\mathbb{R}^{6} \times T^{4}$ [12]. More precisely, we show that the external part on $\mathbb{R}^{6}$ of the hybrid action (plus the action for the bosons $\rho$ and $\sigma$ ) can be replaced by the six-dimensional pure spinor action found in $[7,8]$. The internal part on $T^{4}$ is described in RNS variables and remains the same in the pure spinor formalism.

We proceed as outlined in the introduction and summarized in figure 1. In section 3.1 we rewrite the $\mathcal{N}=2$ hybrid action such that $\mathcal{N}=1$ supersymmetry becomes manifest [13]. In section 3.2 we discuss the corresponding gauge-fixed action. In section 3.3 we will then show that, after an appropriate field redefinition, the gauge-fixed hybrid action turns into the six-dimensional pure spinor action. Finally, in section 3.4 we relate the corresponding BRST operators. 


\subsection{Hybrid formalism with manifest $\mathcal{N}=1$ superspace variables}

The (holomorphic part of the) hybrid action in its original form is given by [12]

$$
S_{\text {hybrid }}=\int d^{2} z\left[\frac{1}{2} \partial x^{m} \bar{\partial} x_{m}+p_{\alpha} \bar{\partial} \theta^{\alpha}\right]+S_{B}+S_{C},
$$

where the external part on $\mathbb{R}^{6}$ is described by the six bosons $x^{m}(m=0, \ldots, 5)$, four fermions $\theta_{\alpha}$ and their conjugates $p^{\alpha}(\alpha=1, \ldots, 4)$ and an action $S_{B}$ for the two chiral bosons $\sigma$ and $\rho$ appearing in the hybrid formalism. The action $S_{C}$ describes the internal part on $T^{4}$ in RNS variables. Here we have assumed that the reader is familiar with the hybrid formalism [12]. A summary of the fields and their properties is given in appendix B.

Unlike in the Green-Schwarz formalism, only half of the usual eight $\theta_{I}^{\alpha}$ variables, say $\theta^{\alpha}=\theta_{2}^{\alpha}$, of six-dimensional $\mathcal{N}=1$ supersymmetry are manifest. However, as suggested in $[13,14]$, it is possible to add $\theta_{1}^{\alpha}$ to the hybrid variables, as well as constraints $D_{\alpha}$, which allow the additional variables $\theta_{1}^{\alpha}$ to be gauged away.

Then, the hybrid action may be written as

$$
S_{\text {hybrid }}=\int d^{2} z\left[\frac{1}{2} \partial x^{m} \bar{\partial} x_{m}+p_{\alpha}^{I} \bar{\partial} \theta_{I}^{\alpha}\right]+S_{B}+S_{C} .
$$

Equivalence to the original hybrid action (3.1) requires that $\theta_{1}^{\alpha}$ and $p_{\alpha}^{1}$ satisfy the first-class constraints [14]

$$
D_{\alpha}=d_{\alpha}^{1}-e^{-\rho-i \sigma} d_{\alpha}^{2}=0,
$$

where $x^{\alpha \beta}=\left(\Gamma_{m}\right)^{\alpha \beta} x^{m}$ and $d_{\alpha}^{I}$ defined as before. Since $D_{\alpha}(z) \theta_{1}^{\beta}(w) \sim \delta_{\alpha}^{\beta}(z-w)^{-1}$, the additional variables $\theta_{1}^{\alpha}$ transform as

$$
\delta \theta_{1}^{\alpha}(w)=\oint d z \varepsilon^{\beta}(z) D_{\beta}(z) \theta_{1}^{\alpha}(w)=\varepsilon^{\alpha}(w)
$$

under the gauge invariance generated by $D_{\alpha}$, as required for superspace variables. The gauge invariance may be used to gauge-fix $\theta_{1}^{\alpha}=0$, in which case (3.2) reduces to (3.1). Note that gauge fixing $\theta_{1}^{\alpha}=0$ does not produce any ghosts since the generating algebra has trivial anticommutation relations.

\subsection{Gauge-fixed hybrid action}

We now implement the constraints of (3.3) into the action (3.2) by introducing Lagrange multipliers $f^{\alpha}$. The extended action then has the form

$$
S_{\text {hybrid }}=\int d^{2} z\left[\frac{1}{2} \partial x^{m} \bar{\partial} x_{m}+p_{\alpha}^{I} \bar{\partial} \theta_{I}^{\alpha}-f^{\alpha} D_{\alpha}\right]+S_{B}+S_{C}
$$

with $D_{\alpha}$ as in (3.3). Both the constraints $D_{\alpha}$ and Lagrange multipliers $f^{\alpha}$ are fields of conformal weight 1 .

On general grounds it can be shown that every constraint induces a gauge symmetry on the extended action [15]. This gauge symmetry is given by

$$
\delta_{D} F(w)=\oint_{C_{w}} d z \epsilon^{\alpha}(z) D_{\alpha}(z) F(w)
$$


for any field $F$ in (3.5) except for the Lagrange multipliers $f^{\alpha}$. The gauge transformation acting on $f^{\alpha}$ can then be defined such that $S_{\text {hybrid }}$ is gauge invariant. The general form of $\delta_{D} f^{\alpha}$ can be found in [15]. Since the constraints anticommute with each other, $\delta_{D} f^{\alpha}$ simplifies a lot to

$$
\delta_{D} f^{\alpha}=\bar{\partial} \epsilon^{\alpha} .
$$

We now gauge-fix this symmetry such that $f^{\alpha}=0 .{ }^{1}$ By the usual Faddeev-Popov method the resulting functional determinant $\Delta_{F P}=\operatorname{det}\left(\bar{\partial} \delta^{2}(z-w) \delta_{\beta}^{\alpha}\right)$ can be rewritten as a functional integral over ghost fields, here $\beta_{\alpha}$ and $\gamma^{\alpha}$. The full action then reads

$$
S_{\text {hybrid }}=\int d^{2} z\left[\frac{1}{2} \partial x^{m} \bar{\partial} x_{m}+p_{\alpha}^{I} \bar{\partial} \theta_{I}^{\alpha}+\beta_{\alpha} \bar{\partial} \gamma^{\alpha}\right]+S_{B}+S_{C}
$$

The fields $\beta_{\alpha}$ and $\gamma^{\alpha}$ are bosonic ghosts of conformal weight 1 and 0 , respectively, and transform in the Weyl representation $\mathbf{4}_{\mathbf{s}}$ of $\mathrm{SO}(6)$.

\subsection{Equivalence of the pure spinor and hybrid actions}

The next step will be to relate the hybrid action in its gauge-fixed form (3.8) to the pure spinor action

$$
S_{\mathrm{ps}}=\int d^{2} z\left[\frac{1}{2} \partial x^{m} \bar{\partial} x_{m}+p_{\alpha}^{I} \bar{\partial} \theta_{I}^{\alpha}+w_{\alpha}^{I} \bar{\partial} \lambda_{I}^{\alpha}\right]+S_{C}
$$

which corresponds to the six-dimensional pure spinor action (2.16) plus a compact part, $S_{C}$, in RNS variables.

The pure spinor constraints in six dimensions require two independent spinors, each in the $\mathbf{4}_{\mathbf{s}}$ of $\mathrm{SO}(6)[7,8]$. For the following it is convenient to temporarily break $\mathrm{SO}(6)$ down to $\mathrm{U}(3)$ such that

$$
\mathbf{4}_{\mathrm{s}} \oplus \mathbf{4}_{\mathrm{s}} \rightarrow \mathbf{3} \oplus \mathbf{3} \oplus \mathbf{1} \oplus \mathbf{1} .
$$

We can write this decomposition under the subgroup $\mathrm{U}(3)$ explicitly as $\lambda^{\alpha}=\left(\lambda^{+}, \lambda^{a}\right)$ with $a=1,2,3$. As it is shown in [8], the pure spinor constraint (2.1) implies

$$
\lambda_{2}^{a}=\frac{\lambda_{2}^{+}}{\lambda_{1}^{+}} \lambda_{1}^{a} .
$$

Therefore one of the $\mathbf{3}$ representations is completely determined by the remaining $\mathbf{3} \oplus \mathbf{1} \oplus \mathbf{1}$ representation, which in turn can be interpreted as the $\mathrm{U}(3)$ invariant representation of pure spinors. This gives exactly five degrees of freedom for the pure spinor, as required.

Using the explicit solution (3.11) of the pure spinor constraint in terms of U(3)invariant representations, the pure spinor part of the action (3.9) can solely be expressed in terms of the five pure spinor degrees of freedom $\lambda_{1}^{+}, \lambda_{1}^{a}$ and $\lambda_{2}^{+}$as

$$
\omega_{\alpha}^{I} \bar{\partial} \lambda_{I}^{\alpha}=\left(\omega_{\alpha}^{1}+\frac{\lambda_{2}^{+}}{\lambda_{1}^{+}} \omega_{\alpha}^{2}\right) \bar{\partial} \lambda_{1}^{\alpha}+\left(\lambda_{1}^{\alpha} \omega_{\alpha}^{2}\right) \bar{\partial}\left(\frac{\lambda_{2}^{+}}{\lambda_{1}^{+}}\right) .
$$

\footnotetext{
${ }^{1}$ Note that this gauge-fixing is different from that of the previous subsection, $\theta^{1}=0$, which led back to $(3.1)$.
} 
We may now deduce the hybrid action in the form (3.8) from the pure spinor action (3.9) using (3.12). It is natural to assume that four of the five pure spinor degrees of freedom, $\lambda_{1}^{\alpha}$, are related to the four ghosts $\gamma^{\alpha}$ in the hybrid formalism. We therefore set

$$
\gamma^{\alpha}=\lambda_{1}^{\alpha}, \quad \beta_{\alpha}=\omega_{\alpha}^{1}+\frac{\lambda_{2}^{+}}{\lambda_{1}^{+}} \omega_{\alpha}^{2}
$$

and the pure spinor action simplifies to

$$
S_{\mathrm{ps}}=\int d^{2} z\left[\frac{1}{2} \partial x^{m} \bar{\partial} x_{m}+p_{\alpha}^{I} \bar{\partial} \theta_{I}^{\alpha}+\beta_{\alpha} \bar{\partial} \gamma^{\alpha}+w \bar{\partial} \lambda\right]+S_{C} .
$$

The variables $\lambda$ and $w$ correspond to the fifth component of the pure spinor and its conjugate momentum and are defined by $\lambda \equiv \frac{\lambda_{2}^{+}}{\lambda_{1}^{+}}$and $w \equiv \lambda_{1}^{\alpha} \omega_{\alpha}^{2}$.

While the first three terms in (3.14) already agree with those of (3.8), the fifth component of the pure spinor still needs to be related to the hybrid variables. A single component of the pure spinor $w \lambda$-system has central charge $c=2$. In the hybrid formalism there are two bosonic ghosts $\sigma$ and $\rho$ with total central charge $c_{\sigma}+c_{\rho}=-26+28=2$. We may therefore conjecture that $\sigma$ and $\rho$ make up the fifth component of the pure spinor. Indeed, as we will show now, they can be obtained by bosonising the $w \lambda$-system in the appropriate way.

The $w \lambda$-system is formally a bosonic ghost system with conformal weights $[\lambda]=0$ and $[w]=1$ and energy-momentum tensor

$$
T^{w \lambda}=(\partial w) \lambda-\partial(w \lambda) .
$$

Each bosonic ghost system can be decomposed into a free boson, which in the following we denote by $\rho$, and an anticommuting $b c$ system. In particular, we may rewrite $w$ and $\lambda$ as

$$
w=e^{\rho} \partial c, \quad \lambda=e^{-\rho} b .
$$

In order to identify $\rho$ with the corresponding ghost in the hybrid formalism, we choose $Q_{\rho}=3$ as the background charge for $\rho$ such that its central charge becomes $c_{\rho}=1+3 Q_{\rho}^{2}=$ 28 , as required. Since, in general, $\left[e^{n \rho}\right]=-\frac{n^{2}}{2}+\frac{n}{2} Q_{\rho}$ for the conformal weight of $e^{n \rho}$, we get $\left[e^{\rho}\right]=1$ and $\left[e^{-\rho}\right]=-2$, and therefore $[b]=2$ and $[c]=-1$, which are the conformal weights of a standard $b c$ system.

As usual, one can go further and bosonise the $b c$ system as $b=e^{-i \sigma}$ and $c=e^{i \sigma}$, with central charge given by $c_{\sigma}=1-3 Q_{\sigma}^{2}=-26$ for $Q_{\sigma}=3$. The conformal weights are $\left[e^{i n \sigma}\right]=\frac{n^{2}}{2}-\frac{n}{2} Q_{\sigma}$. The energy-momentum tensor (3.15) can then be rewritten in terms of $\rho$ and $\sigma$ as

$$
T^{\rho, \sigma}=-\frac{1}{2} \partial \rho \partial \rho-\frac{1}{2} \partial \sigma \partial \sigma-\frac{3}{2} \partial^{2}(\rho+i \sigma),
$$

which is identical to the energy-momentum tensor corresponding to the ghost action $S_{B}$ in the hybrid formalism [12]. The term $\int d^{2} z w \bar{\partial} \lambda$ in (3.14) is therefore identical to the ghost action $S_{B}$ in the hybrid formalism. The action (3.14) is thus equivalent to the hybrid action (3.8), $S_{\mathrm{ps}}=S_{\text {hybrid }}$. 


\subsection{Comments on the BRST operators}

As a critical $\mathcal{N}=2$ theory, the hybrid string can be reformulated as a $\mathcal{N}=4$ topological string theory $[12,16]$. Recall that every $\mathcal{N}=2$ superconformal theory with central charge $c=6$ gives rise to a critical $\mathcal{N}=4$ superconformal field theory. The corresponding $\mathcal{N}=4$ algebra is generated by the energy momentum tensor $T$, four fermionic currents $G^{ \pm}$and $\widetilde{G}^{ \pm}$, and three $\mathrm{SU}(2)$ currents $J^{a}(a=1,2,3)$. These currents can be defined from the $\mathcal{N}=2$ currents $\left[T, G^{+}, G^{-}, J\right]$ by $T, G^{+}, G^{-}, \widetilde{G}^{+} \equiv\left[e^{-\int J}, G^{+}\right], \widetilde{G}^{-} \equiv\left[e^{\int J}, G^{-}\right]$, and $J, e^{\int J}, e^{-\int J}$. Explicit expressions for these generators with manifest six-dimensional superspace variables can be found in [13].

Open $\mathcal{N}=4$ string physical vertex operators in hybrid string theory satisfy the physical state conditions ${ }^{2,3}$

$$
G_{0}^{+} \Phi=\widetilde{G}_{0}^{+} \Phi=\left(J_{0}-1\right) \Phi=0, \quad \delta \Phi=G_{0}^{+} \widetilde{G}_{0}^{+} \Lambda^{-} .
$$

Since the cohomology of $\widetilde{G}_{0}^{+}$is trivial $[12,16], \Phi$ can be written as

$$
\Phi=\widetilde{G}_{0}^{+} V, \quad G_{0}^{+} \widetilde{G}_{0}^{+} V=J_{0} V=0
$$

with gauge invariance $\delta V=G_{0}^{+} \Lambda+\widetilde{G}_{0}^{+} \widetilde{\Lambda}$. This gauge invariance can be fixed such that

$$
G_{0}^{-} \Phi=\widetilde{G}_{0}^{-} \Phi=T_{0} \Phi=0
$$

is automatically satisfied [12].

We now need to take into account that we introduced additional $\theta$ variables and added the Lagrange multiplier term $f^{\alpha} D_{\alpha}$. The gauge-fixing $f^{\alpha}=0$ requires us to impose a further condition on the physical states. Since $D_{\alpha}(z) D_{\beta}(w) \sim 0$, the gauge symmetries generated by $D_{\alpha}$ are abelian such that the additional condition has the simple form $\gamma^{\alpha} D_{\alpha}$. After gauge-fixing, a physical state must also satisfy

$$
Q_{\text {hybrid }} \Phi=0
$$

where

$$
\begin{aligned}
Q_{\text {hybrid }} & =\oint d z\left(\gamma^{\alpha} D_{\alpha}\right) \\
& =\oint d z\left(\gamma^{\alpha} d_{\alpha}^{1}-e^{-\rho-i \sigma} \gamma^{\alpha} d_{\alpha}^{2}\right) .
\end{aligned}
$$

In the second line we used the definition of $D_{\alpha}$ given by (3.3). For the following it is useful to change the minus sign in (3.22) into a plus sign by exploiting the symmetry $\theta_{2} \rightarrow-\theta_{2}$ and $d^{2} \rightarrow-d^{2}$, see [8].

The operator (3.22) can be shown to be equivalent to the BRST operator of the pure spinor formalism. For that, we use the field redefinition of the previous subsection,

$$
\lambda_{1}^{\alpha}=\gamma^{\alpha}, \quad \lambda=\lambda_{2}^{+} / \lambda_{1}^{+}=e^{-\rho-i \sigma},
$$

\footnotetext{
${ }^{2} G_{0}^{+}$is the charge (zero mode) corresponding to the current $G^{+}$, etc.

${ }^{3}$ Analogous conditions hold for the closed superstring [12].
} 
or, equivalently, by eq. (3.11),

$$
\begin{aligned}
& \lambda_{1}^{\alpha}=\gamma^{\alpha}, \\
& \lambda_{2}^{\alpha}=e^{-\rho-i \sigma} \gamma^{\alpha} .
\end{aligned}
$$

Substituting this into (3.22), we get

$$
Q_{\text {hybrid }}=\oint d z \lambda_{I}^{\alpha} d_{\alpha}^{I}=Q_{\mathrm{ps}}
$$

which is exactly the pure spinor BRST operator $Q_{\mathrm{ps}}$, as defined in $[7,8]$. Recall that nilpotence of $Q_{\mathrm{ps}}$ is ensured by the pure spinor constraint $(2.1)$ [7,8].

We close with a few comments on the vertex operators in both theories. Let us restrict to the massless open string vertex operator which is independent of the compactification variables. In hybrid string theory this operator is obtained by solving the physical state conditions (3.18) and the 'harmonic' BRST-like condition (3.21). As found in [13, 14], such an operator describes the six-dimensional on-shell degrees of freedom of six-dimensional $\mathcal{N}=1$ super Yang-Mills theory. The corresponding integrated vertex operator is given by ${ }^{4}$

$$
\Phi_{\text {hybrid }}=\int d z\left[\Pi^{m} A_{m}+\partial \theta_{I}^{\alpha} A_{\alpha}^{I}+d_{\alpha}^{I} W_{I}^{\alpha}+\frac{1}{2}\left(\gamma^{\alpha}\left(\Gamma^{m n}\right)_{\alpha}^{\beta} \beta_{\beta}\right) F_{m n}\right]
$$

where $\Pi^{m}$ are the superspace momenta, $W_{I}^{\alpha}$ the superspace spinor field strengths and $A_{m}$, $A_{\alpha}^{I}$ the superspace gauge fields $[13,14] . F_{m n}$ is a superspace field strength whose lowest component is the gluon field strength. Each field depends on the superspace coordinates $\left(x^{m}, \theta_{I}^{\alpha}\right)$. Vertex operators of this form were first discussed in ten dimensions in [17].

The vertex operator $\Phi_{\text {hybrid }}$ needs to be compared with the corresponding vertex operator in the pure spinor formalism. It is important to note that, as for the hybrid string, we need to impose both the pure spinor BRST condition (3.25), $Q_{\mathrm{ps}} \Phi=0$, as well as the physical state conditions (3.18), now rewritten in terms of pure spinor variables. The condition (3.25) alone does not put the theory on-shell.

Consider for instance the massless compactification-independent open string vertex operator which is obtained by solving $Q_{\mathrm{ps}} \Phi=0$. In six dimensions it has the form

$$
\Phi_{\mathrm{ps}}=\lambda_{I}^{\alpha} A_{\alpha}^{I}\left(x, \theta_{I}^{\alpha}\right),
$$

where the ghost-number one spinor superfield $A_{\alpha}^{I}$ contains the Yang-Mills degrees of freedom. As repeatedly stated $[4,7], Q_{\mathrm{ps}}$ only selects the off-shell field content of sixdimensional $\mathcal{N}=1$ super-Yang-Mills. $Q_{\mathrm{ps}} \Phi=0$ implies

$$
\lambda_{I}^{\alpha} \lambda_{J}^{\beta} D_{\alpha}^{I} A_{\beta}^{J}=0,
$$

where $D_{\alpha}^{I}=\frac{\partial}{\partial \theta_{I}^{\alpha}}+\frac{1}{2} \varepsilon^{I J}\left(\gamma^{m} \theta_{J}\right)_{\alpha} \frac{\partial}{\partial x^{m}}$.

Since $\lambda_{I}^{\alpha} \lambda_{J}^{\beta}$ is a symmetric tensor under the exchange of $(I, \alpha)$ and $(J, \beta)$, it projects $D_{\alpha}^{I} A_{\beta}^{J}$ onto its symmetric part under this involution. This part decomposes as

$$
D_{\alpha}^{I} A_{\beta}^{J}+D_{\beta}^{J} A_{\alpha}^{I}=\varepsilon^{I J} \Gamma_{\alpha \beta}^{m} A_{m}+\cdots,
$$

\footnotetext{
${ }^{4}$ The last term involving the ghost $\gamma^{\alpha}$ and its conjugate $\beta_{\alpha}$ was later added in [1], see footnote 3 therein.
} 
i.e. into a vector $A_{m}$ and other $n$-form contributions indicated by ellipses. Note here that in six dimensions a general antisymmetric bispinor $f_{\alpha \beta}(\alpha, \beta=1, \ldots, 4)$ is related to a vector $A_{m}(m=0, \ldots, 5)$ by $f_{\alpha \beta}=\Gamma_{\alpha \beta}^{m} A_{m}$. By substituting this into (3.28) and using the pure spinor constraint (2.3), one can show that all $n$-form contributions vanish, i.e. all terms in the ellipses in (3.29) are zero. Then, (3.29) becomes exactly the linearised constraint $F_{\alpha \beta}^{I J}=0$, which is imposed on the superspace field strength $F_{\alpha \beta}^{I J}$, cf. with eq. (3.17) in [18]. Unlike in ten dimensions, this constraint is off-shell since one cannot deduce the equation of motions from it. (Since $Q_{\text {hybrid }}=Q_{\mathrm{ps}}$ this implies that also $Q_{\text {hybrid }}$ selects only the off-shell field content.) It is therefore natural to assume that the conditions (3.18) put the pure spinor theory on-shell, as it does in hybrid string theory. ${ }^{5}$

In conclusion, we have shown that for superstring theory on $\mathbb{R}^{6} \times T^{4}$ a gauge-fixed version of the hybrid string is related to a (hybrid) pure spinor string theory by a simple field redefinition given by eq. (3.24). In particular, this transformation identifies both the actions as well as the hybrid string BRST-like condition (3.22) and the pure spinor BRST operator (3.25). These BRST operators determine the off-shell field content of $\mathcal{N}=1$ sixdimensional super Yang-Mills. We argued that in order to put the hybrid pure spinor theory on-shell a physical vertex operator also has to satisfy the conditions (3.18). Of course, for this the physical state conditions (3.18) must be rewritten in pure spinor variables using the identifications (3.24), which we have not done explicitly. We are fairly optimistic though that the conditions (3.18) will then provide the required equations of motion for the gauge field $A_{\alpha}^{I}\left(x, \theta_{I}^{\alpha}\right)$, as it does for the corresponding hybrid string vertex operator. The hybrid version of the pure spinor string then provides the appropriate framework for six-dimensional pure spinors.

An open question is the relation of the four-dimensional pure spinor action to the corresponding hybrid string on $\mathbb{R}^{4} \times T^{6}[20]$. Here we encounter a puzzle [11]: The central charge of the pure spinor theory parameterizing the part on $\mathbb{R}^{4}$ is zero $[7,8]$. If we wish to describe the six internal directions in RNS variables, we obtain a (topological) $\mathcal{N}=2$ string with $\hat{c}=3(c=9)$. However, a critical $\mathcal{N}=2$ string has $\hat{c}=2(c=6)$, and the pure spinor theory cannot be related to hybrid strings in a simple way. Possibly such a naive compactification of the pure spinor theory describes the BPS sector of the compactified superstring [3]. More work is needed here to make the relation precise.

\section{Acknowledgments}

We would like to thank Matthias Gaberdiel, Stefan Hohenegger, Peter Rønne, Volker Schomerus and Niclas Wyllard for useful discussions related to this work. We are also grateful to Nathan Berkovits for helpful comments on a preliminary version of this paper. This research has been supported by the Swiss National Science Foundation.

\footnotetext{
${ }^{5} \mathrm{~A}$ different method to put the pure spinor theory on-shell was proposed in [19].
} 


\begin{tabular}{|c|c|c|c|c|}
\hline RNS & & $c$ & $h$ & $\varepsilon$ \\
\hline 6 bosons & $x^{m}$ & 6 & 1 & -1 \\
6 fermions & $\psi^{m}$ & 3 & $\frac{1}{2}$ & +1 \\
\hline 2 bosons & $\beta, \gamma$ & 11 & $\frac{3}{2},-\frac{1}{2}$ & -1 \\
2 fermions & $b, c$ & -26 & $2,-1$ & +1 \\
\hline$T^{4}$ & $Y^{i}, \eta^{i}$ & 6 & $1, \frac{1}{2}$ & $\mp 1$ \\
\hline
\end{tabular}$\quad$\begin{tabular}{|c|c|c|c|c|c|}
\hline hybrid & & $c$ & $h$ & $\varepsilon$ \\
\hline 6 bosons & $x^{m}$ & 6 & 1 & -1 \\
8 fermions & $p_{\alpha}, \theta^{\alpha}$ & -8 & 1,0 & +1 \\
\hline 1 boson & $\rho$ & 28 & 0 & -1 \\
1 boson & $\sigma$ & -26 & 0 & +1 \\
\hline$T^{4}$ & $Y^{i}, \eta^{i}$ & 6 & $1, \frac{1}{2}$ & $\mp 1$ \\
\hline
\end{tabular}

$\downarrow$

\begin{tabular}{|c|c|c|c|c|}
\hline pure spinor & & $c$ & $h$ & $\varepsilon$ \\
\hline 6 bosons & $x^{m}$ & 6 & 1 & -1 \\
16 fermions & $p_{\alpha}^{I}, \theta_{I}^{\alpha}$ & -16 & 1,0 & +1 \\
\hline 5 pure spinors & $\lambda_{I}^{\alpha}, w_{\alpha}^{I}$ & 10 & 0,1 & -1 \\
\hline$T^{4}$ & $Y^{i}, \eta^{i}$ & 6 & $1, \frac{1}{2}$ & $\mp 1$ \\
\hline
\end{tabular}

Table 1. Overview of the fields in the RNS, hybrid and pure spinor formalism of string theory on $\mathbb{R}^{6} \times T^{4} . c$ and $h$ denote the contribution to the total central charge and the conformal weight of the fields. The value $\varepsilon=+1(\varepsilon=-1)$ refers to Fermi (Bose) statistics.

\section{A Six-dimensional gamma matrices}

We give an explicit realisation of the matrices $\Gamma_{\alpha \beta}^{m}$ used throughout this paper satisfying (2.2). They can be chosen to be

$$
\begin{array}{ll}
\Gamma^{0}=-i \mathbb{1} \otimes \tau^{2} & \Gamma^{3}=\tau^{2} \otimes \tau^{3} \\
\Gamma^{1}=\tau^{2} \otimes \tau^{1} & \Gamma^{4}=-i \tau^{2} \otimes \mathbb{1} \\
\Gamma^{2}=i \tau^{1} \otimes \tau^{2} & \Gamma^{5}=-i \tau^{3} \otimes \tau^{2}
\end{array}
$$

where $\tau^{i}$ are the usual two-dimensional Pauli matrices. The Weyl indices are raised by the epsilon tensor according to the rule

$$
\left(\Gamma^{m}\right)^{\alpha \beta}=\frac{1}{2} \varepsilon^{\alpha \beta \gamma \delta} \Gamma_{\gamma \delta}^{m} .
$$

A useful identity is

$$
\left(\Gamma^{m}\right)_{\alpha \beta}\left(\Gamma_{m}\right)_{\gamma \delta}=-2 \varepsilon_{\alpha \beta \gamma \delta} .
$$

\section{B Summary of fields in RNS, hybrid and pure spinor formalism}

In this appendix we summarize the fields occurring in the three worldsheet formalisms of string theory on $\mathbb{R}^{6} \times T^{4}$.

In the RNS formalism the external part on $\mathbb{R}^{6}$ is described in terms of six bosons and fermions, $x^{m}$ and $\psi^{m}(m=0, \ldots, 5)$, while the internal part on $T^{4}$ is parameterized by four bosons and fermions, $Y^{i}$ and $\eta^{i}(i=1, \ldots, 4)$. The ghost sector is given by the standard $b c$ and $\beta \gamma$ systems. These fields and their properties are listed in the upper left box of table 1. 
The hybrid formalism is obtained from the RNS formalism [12] by first embedding the critical $\mathcal{N}=1$ RNS string into a critical $\mathcal{N}=2$ string and then, after twisting, performing the following field redefinition. The bosons $x^{m}$ are the same as in the RNS string. The fermions and ghosts are reorganised into eight fermions $p_{\alpha}, \theta^{\alpha}(\alpha=1, \ldots, 4)$ and two chiral bosons, $\sigma$ and $\rho$. The latter are obtained by bosonising both the $b c$ as well as the $\beta \gamma$ system in the standard way, i.e. as $(b, c)=\left(e^{-i \sigma}, e^{i \sigma}\right)$ and $(\beta, \gamma)=\left(e^{-\phi+\kappa} \partial \kappa, e^{\phi-\kappa}\right)$. The corresponding background charges are $Q_{\kappa}=1, Q_{\phi}=2$ and $Q_{\sigma}=3\left(\varepsilon_{\kappa}=-\varepsilon_{\phi}=1\right)$. Then $\sigma$ and $\rho$ are defined by

$$
\partial \sigma=i b c, \quad \rho=-2 \phi-i \kappa-i H_{C}^{R N S},
$$

where $H_{C}^{R N S}=H_{4}+H_{5}$ are the bosonised fermions of $T^{4}$. Both fields are spacetime bosons of conformal weight zero but have opposite statistics, $\varepsilon_{\sigma}=-\varepsilon_{\rho}=1$. Their contribution to the central charge is $c=1-\varepsilon 3 Q^{2}$ with background charges $Q_{\sigma}=Q_{\rho}=3$, and therefore $c_{\sigma}=-26$ and $c_{\rho}=28$. The fermions $p_{\alpha}, \theta^{\alpha}$ are defined in terms of the RNS variables as

$$
\begin{aligned}
\theta^{\alpha} & =\left[e^{\frac{1}{2} \phi} \Sigma^{+++++}, e^{\frac{1}{2} \phi} \Sigma^{--+++}, e^{\frac{1}{2} \phi} \Sigma^{-+-++}, e^{\frac{1}{2} \phi} \Sigma^{+--++}\right], \\
p_{\alpha} & =\left[e^{-\frac{1}{2} \phi} \Sigma^{------}, e^{-\frac{1}{2} \phi} \Sigma^{++---}, e^{-\frac{1}{2} \phi} \Sigma^{+-+--}, e^{-\frac{1}{2} \phi} \Sigma^{-++--}\right],
\end{aligned}
$$

where $\phi$ is the $\beta \gamma$-boson and $\Sigma^{\alpha}$ is the spin field of conformal weight $\frac{5}{8}$ defined by

$$
\Sigma^{\alpha}=e^{\frac{i}{2} \sum_{I=1}^{5} \epsilon_{I} H_{I}}
$$

with $\epsilon_{I}= \pm 1$. The bosons $H_{1,2,3}$ and $H_{4,5}$ are obtained by bosonising the fermions $\psi^{m}$ and $\eta^{i}$, respectively. Since $e^{n \phi}$ has weight $-\frac{n^{2}}{2}-n$, which is $-\frac{5}{8}$ and $\frac{3}{8}$ for $e^{ \pm \phi / 2}, p_{\alpha}$ and $\theta^{\alpha}$ form four (fermionic) $b c$-systems with weights $(1,0)$. Their contribution to the central charge is $c=4 \cdot(-2)=-8{ }^{6}$ The internal part on $T^{4}$ is the same as in the RNS formalism. The fields of the hybrid formalism are summarized in the upper right box of table $1 .{ }^{7}$

The pure spinor formalism requires again six bosons $x^{m}$ and now sixteen fermions $p_{\alpha}^{I}$ and $\theta_{I}^{\alpha}(I=1,2 ; \alpha=1, \ldots, 4)$, twice as many as in the hybrid formalism. The pure spinor part consists of the fields $\lambda_{I}^{\alpha}, w_{\alpha}^{I}$, which because of the pure spinor condition formally represent five (bosonic) $\beta \gamma$-systems with weights $(0,1)$. Their contribution to the central charge is therefore $c=5 \cdot 2=10$. The internal part is again as in the RNS formalism. The connection between the hybrid and the pure spinor formalism is described in section 3 . The fields and their properties are shown in the lower box of table 1.

Open Access. This article is distributed under the terms of the Creative Commons Attribution Noncommercial License which permits any noncommercial use, distribution, and reproduction in any medium, provided the original author(s) and source are credited.

\footnotetext{
${ }^{6}$ For $b c(\beta \gamma)$ systems the anomaly contribution of the ghosts is $c=-2 \varepsilon(6 \lambda(\lambda-1)+1)$, where $\lambda=h_{b}$ $\left(h_{\beta}\right)$ and $\varepsilon=1(-1)$.

${ }^{7}$ The table lists only the matter part of the $\mathcal{N}=2$ hybrid string. Note that the critical central charge for the matter part of an $\mathcal{N}=2$ string is $c=6$.
} 


\section{References}

[1] N. Berkovits, Super-Poincaré covariant quantization of the superstring, JHEP 04 (2000) 018 [hep-th/0001035] [SPIRES].

[2] N. Berkovits, Multiloop amplitudes and vanishing theorems using the pure spinor formalism for the superstring, JHEP 09 (2004) 047 [hep-th/0406055] [SPIRES].

[3] N. Berkovits, Pure spinor formalism as an $N=2$ topological string, JHEP 10 (2005) 089 [hep-th/0509120] [SPIRES].

[4] N. Berkovits, ICTP lectures on covariant quantization of the superstring, hep-th/0209059 [SPIRES].

[5] Y. Oz, The pure spinor formulation of superstrings, Class. Quant. Grav. 25 (2008) 214001 [arXiv:0910.1195] [SPIRES].

[6] O.A. Bedoya and N. Berkovits, GGI lectures on the pure spinor formalism of the superstring, arXiv:0910.2254 [SPIRES].

[7] P.A. Grassi and N. Wyllard, Lower-dimensional pure-spinor superstrings, JHEP 12 (2005) 007 [hep-th/0509140] [SPIRES].

[8] N. Wyllard, Pure-spinor superstrings in D =2,4,6, JHEP 11 (2005) 009 [hep-th/0509165] [SPIRES].

[9] I. Adam, P.A. Grassi, L. Mazzucato, Y. Oz and S. Yankielowicz, Non-critical pure spinor superstrings, JHEP 03 (2007) 091 [hep-th/0605118] [SPIRES].

[10] I. Adam, A. Dekel, L. Mazzucato and Y. Oz, Integrability of type-II superstrings on Ramond-Ramond backgrounds in various dimensions, JHEP 06 (2007) 085 [hep-th/0702083] [SPIRES].

[11] N. Berkovits, Explaining the pure spinor formalism for the superstring, JHEP 01 (2008) 065 [arXiv:0712.0324] [SPIRES].

[12] N. Berkovits, C. Vafa and E. Witten, Conformal field theory of AdS background with Ramond-Ramond flux, JHEP 03 (1999) 018 [hep-th/9902098] [SPIRES].

[13] N. Berkovits, Quantization of the type-II superstring in a curved six-dimensional background, Nucl. Phys. B 565 (2000) 333 [hep-th/9908041] [SPIRES].

[14] N. Berkovits, Quantization of the superstring in Ramond-Ramond backgrounds, Class. Quant. Grav. 17 (2000) 971 [hep-th/9910251] [SPIRES].

[15] M. Henneaux and C. Teitelboim, Quantization of gauge systems, Princeton University Press, Princeton U.S.A. (1992), pag. 520 [SPIRES].

[16] J. Kappeli, S. Theisen and P. Vanhove, Hybrid formalism and topological amplitudes, hep-th/0607021 [SPIRES].

[17] W. Siegel, Classical superstring mechanics, Nucl. Phys. B 263 (1986) 93 [SPIRES].

[18] P.S. Howe, G. Sierra and P.K. Townsend, Supersymmetry in six-dimensions, Nucl. Phys. B 221331 (1983) 331 [SPIRES].

[19] M. Cederwall and B.E.W. Nilsson, Pure spinors and D $=6$ Super-Yang-Mills, arXiv:0801.1428 [SPIRES].

[20] N. Berkovits, Covariant quantization of the Green-Schwarz superstring in a Calabi-Yau background, Nucl. Phys. B 431 (1994) 258 [hep-th/9404162] [SPIRES]. 